\title{
Relationship among Discharges of Neighboring Neurons in the Rat Prefrontal Cortex During Spatial Working Memory Tasks
}

\author{
Min Whan Jung, ${ }^{1,3}$ Yulin Qin, ${ }^{5}$ Daeyeol Lee, ${ }^{6}$ and Inhee Mook-Jung ${ }^{2,4}$ \\ ${ }^{1}$ Neuroscience Laboratory, Institute for Medical Sciences, ${ }^{2}$ Brain Disease Research Center, ${ }^{3}$ Department of Physiology, and \\ ${ }^{4}$ Department of Anatomy, Ajou University School of Medicine, Suwon 442-721, Korea, ${ }^{5}$ Department of Physical Medicine \\ and Rehabilitation, Baylor College of Medicine, Houston, Texas 77030, and ${ }^{6}$ Department of Brain and Cognitive Sciences \\ and Center for Visual Science, University of Rochester, Rochester, New York 14627
}

The relationship among discharges of neurons that were recorded simultaneously with tetrodes in the rat medial prefrontal cortex was analyzed. Spatial working memory tasks were divided into several distinct stages based on the behavioral correlates of individual neurons, and interneuronal correlation of signal (mean discharge rate at each stage) and noise (trial-to-trial deviation from the signal) was calculated. Behavioral correlates of neighboring neurons were quite heterogeneous and, accordingly, average signal correlation was relatively low $(\sim 0.16)$. Noise correlation was even lower $(\sim 0.06)$, but neuronal noise was more correlated among the neurons with similar signals. Spikes under- lying the signal and noise correlation among the prefrontal cortical neurons were loosely synchronized over a few hundred milliseconds. These results suggest that neighboring prefrontal cortical neurons process largely independent information and have weakly correlated noise and that precisely synchronized spikes play a relatively minor role in producing the correlated signal and noise among these neurons.

Key words: single unit; signal correlation; noise correlation; cortical column; adjacent neurons; prefrontal cortex; synchronous firing; tetrode
Cortical neurons typically show variable discharges in response to repeated presentations of an identical stimulus (Rieke et al., 1997). This leads to an intuitive hypothesis that cortical information processing should be redundant to achieve reliable transmission of signals. Consistent with this idea, the cortex is organized in such a way that neurons within the same cortical column tend to share common inputs (Eccles, 1981). Physiological studies also have shown that neurons within the same cortical column have similar response characteristics (Mountcastle, 1957). Redundant signal processing is advantageous in several respects. Signal-to-noise ratio can be enhanced by averaging signals across many noisy neurons, and time-varying signals can be estimated reliably in a short time period by reducing the need for temporal integration (Shadlen and Newsome, 1998). In addition, signals can be transmitted reliably even with a partial damage or discharge failure. The price for redundancy is a reduced capacity for information processing, however. The maximum information processing capacity will be seriously limited if a large fraction of neurons process identical information. These considerations suggest that there are two opposing evolutionary pressures for the cortical circuits, i.e., redundancy and independence (Linsker, 1988). What level of redundant information would be optimal? It is conceivable that this depends on such factors as the number of neurons, connectivity, and the nature of computation performed by a given network of neurons. Depending on whether such factors remain constant across different cortical areas, the level of redundant information processing may vary across different regions of the cortex, or different cortical regions may display a similar level of redundancy.

Received Sept. 10, 1999; revised May 24, 2000; accepted May 24, 2000.

This work was supported by the Korea Ministry of Science and Technology under the Brain Science Research Program and the Korea Science and Engineering Foundation grant through the Brain Disease Research Center at Ajou University (M.W.J.), United States Public Health Service Grants MH46823 (B.M.) and MH59216 (D.L.), and the McDonnell-Pew Cognitive Neuroscience Grant 99-27 from the James S. McDonnell Foundation (D.L.). We thank Drs. Bruce McNaughton and Carol Barnes for their help during collection of single-unit data and Yunbok Kim and Eun Ha Baeg for their help with analysis.

Correspondence should be addressed to Dr. Min Whan Jung, Neuroscience Laboratory, Institute for Medical Sciences, Ajou University, Suwon 442-721, Korea. E-mail: min@madang.ajou.ac.kr.

Copyright (C) 2000 Society for Neuroscience $0270-6474 / 00 / 206166-07 \$ 15.00 / 0$
Another important issue related to cortical information processing is correlated noise in the rate of neuronal discharges. On one hand, correlated noise has profound effects on response pooling. In a simple situation in which all neurons process identical signals, correlated noise plays a negative role of limiting maximum enhancement of signal-to-noise ratio (Johnson et al., 1973; Zohary et al., 1994), whereas it will be cancelled out among neurons that process opposite signals (van Kan et al., 1985; Lee et al., 1998b). On the other hand, information can be transmitted by consistent relationship among two or more neurons so that they transmit information synergistically. Depending on its relationship with signal correlation, correlated noise may lead to either redundant or synergistic information transmission (Panzeri et al., 1999). Correlated noise can be measured experimentally by comparing response profiles of simultaneously recorded neuron pairs. At present, however, there exist only a handful of studies that measured correlated noise quantitatively (van Kan et al., 1985; Gawne and Richmond, 1993; Zohary et al., 1994; Gawne et al., 1996; Lee et al., 1998a; Maynard et al., 1999). To address the degree of redundant information processing and correlated noise in the prefrontal cortex (PFC), we examined relationship among discharges of neighboring PFC neurons that were recorded with tetrodes in rats performing spatial working memory tasks.

Behavioral correlates of these individual units have been reported previously (Jung et al., 1998).

\section{MATERIALS AND METHODS}

Unit recording. Single units were recorded in deep layers (4-6) of the medial PFC of four young ( 9 month) male Fischer-344 rats. Animal care and surgery were performed following the National Institutes of Health guidelines. Recordings were made using tetrodes in the prelimbic cortex, infralimbic cortex, frontal area 2, and anterior dorsal cingulate gyrus. Tetrodes consist of a bundle of four twisted electrode wires that allow both simultaneous recordings of multiple neurons and identification of each individual unit based on spike amplitude profile of each unit activity recorded across four electrode channels (Recce and O'Keefe, 1989; Wilson and McNaughton, 1993). This technique, compared to other methods, has a number of advantages in recording multiple neighboring neurons with clear isolation of individual units (Gray et al., 1995). Detailed description of recording procedures has been reported (Jung et al., 1998). Briefly, unit signals from the tetrode were recorded via an FET source-follower headstage mounted on the animal's head. Output signals from the headstage were filtered between 0.6 and $6 \mathrm{kHz}$, digitized at $32 \mathrm{kHz}$, and stored on 

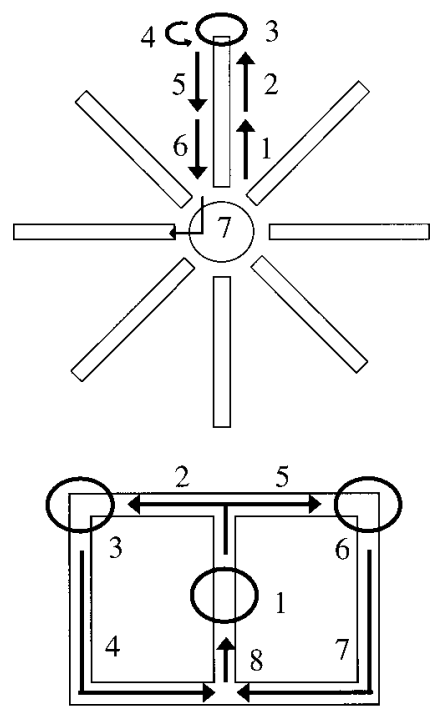

Figure 1. Behavioral tasks and behavioral stages. Top, Eight-arm radial maze, spatial working memory task. The rat had to visit the end of each arm not previously visited on the current trial to obtain chocolate milk reward. The task was divided into several behavioral stages as shown: 1, running outward, 2, approaching goal; 3, staying at goal; 4, turning; 5, running inward (distal); 6 , running inward (proximal); and 7, selecting a new arm. Corresponding parts on all eight arms were combined as one stage. Intertrial interval is an additional behavioral stage that is not shown in the figure. The average duration of each stage was $15.8(1), 8.5(2), 53.4(3), 1.5$ (4), $6.1(5), 6.5(6), 59.3$ (7), and $45.4 \mathrm{sec}$ (intertrial interval). Bottom, Figure eight maze, delayed spatial alternation task. Starting from the center of the maze, the animal had to alternately visit two reward sites to obtain chocolate morsels. Behavioral stages were divided as shown: 1, consuming reward at center; 2, approaching left goal; 3, consuming reward at left goal; 4 , returning from left goal; 5, approaching right goal; 6 , consuming reward at right goal, 7 , returning from right goal, and 8 , approaching center. The average duration of each stage was $4.4(1), 2.2$ (2), 4.4 (3), 4.3 (4), 3.0 (5), $4.3(6), 4.1(7)$, and $0.9 \sec (8)$.

80486 personal computers. The data were transferred to a SUN 4 workstation, and units were isolated by projecting the four-channel relative amplitude data two-dimensionally and applying boundaries to each subjectively identified unit cluster. To prevent potential overlap of clusters, only those pairs of clusters that were completely nonoverlapping in at least one projection were included in the analysis.

A previous estimate suggested that each channel of a tetrode records spike signals from locations no further than $65 \mu \mathrm{m}$ (Gray et al., 1995; same tetrodes were used as in the present study). Thus, overall, a tetrode records from a volume of tissue with a diameter of $\sim 150 \mu \mathrm{m}$. The size of columns in the PFC ranged from 230 to $1190 \mu \mathrm{m}$ in monkeys (Goldman-Rakic and Schwartz, 1982; Bugbee and Goldman-Rakic, 1983). The median values were between 550 and $680 \mu \mathrm{m}$ in adults and 400 and $500 \mu \mathrm{m}$ in infants. To our knowledge, no such data are available for the rat PFC. Assuming that the rat PFC contains about the same size of columns as those of monkeys, the range of tetrode recording is comparable to the dimensions of cortical columns.

Behavioral tasks. Unit recordings were made while the animals were performing two different spatial working memory tasks. One was an eight-arm radial maze, spatial working memory task and the other was a figure eight maze, delayed spatial alternation task. For the eight-arm maze task, the rat had to visit the end of each arm not previously visited on the current trial to obtain a chocolate milk reward (Fig. 1, top). The rats typically ran 10 trials in $20-30 \mathrm{~min}$. For the delayed spatial alternation task on a figure eight maze, starting from the center of the maze, the animal had to visit alternately two reward sites to obtain chocolate morsels (Fig. 1, bottom). The animal had to come back to the center from a reward site before visiting the other reward site. The rat consumed chocolate morsels at the maze center for 3-7 sec, which served as a delay period. The animal ran a minimum of 15 trials consecutively for each recording session.

Data analysis. Discharges of individual PFC units were generally correlated to distinct stages of the eight-arm maze task and the figure eight maze task (Jung et al., 1998). Based on these behavioral correlates, the behavioral tasks were divided into several stages as indicated in Figure 1. The other behavioral stage during the radial maze task that is not shown in the figure was the intertrial interval.

Because our interest in this study was to examine the discharge patterns of neighboring PFC neurons, only neuron pairs that were simultaneously recorded with a single tetrode were included in the analysis. "Signal" was defined as the mean discharge rate at each stage over multiple trials (usually 10 for radial maze and 15 for figure eight maze) and "noise" was defined as trial-to-trial deviation of firing rates from the mean at each stage (Gawne and Richmond, 1993; Lee et al., 1998a). When calculating noise correlation, spike counts were converted to $z$ scores using the mean and SD for each behavioral stage to remove the influence of different signal strength (Zohary et al., 1994). For the radial maze, corresponding parts on all eight arms were combined as one stage, because most PFC units showed radially symmetrical firing patterns. Correlation coefficients $(r)$ for signal (signal correlation) and noise (noise correlation) were separately calculated and transformed to Fisher's $z$ for normalization of distribution as follows (Snedecor and Cochran, 1989):

$$
z=0.5[\ln (1+r)-\ln (1-r)] .
$$

Statistical significance of signal and noise correlation was determined by recalculating the corresponding correlation coefficient after responses (i.e., firing rates at each stage in a given trial) of one neuron in a given pair were randomly shuffled relative to the responses of the other neuron. For signal correlation, the entire responses of one neuron were shuffled randomly, and for noise correlation, responses were shuffled within each behavioral stage so that a response in one stage of one neuron cannot be paired to a response of the other neuron in a different stage after shuffling. For the original correlation to be significant, it should be consistently higher than the correlation obtained after shuffling.

As reported in Results, interneuronal signal correlation was relatively low in the PFC. There are at least two potential reasons for such low signal correlation. One possibility is that the signal carried by a given neuron is weak and unreliable. Alternatively, signals of individual neurons may be robust, but they are simply not correlated across different neurons. To distinguish between these two possibilities, each behavioral stage defined above was divided into two halves, and correlation coefficient was calculated between the discharge rates of these two epochs. This correlation coefficient should approach 1.0 if the discharge rate across these two epochs is highly correlated (i.e., robust signal). All correlation coefficients were converted to Fisher's $z$ scores for hypothesis testing. All data are expressed as mean \pm SEM.

PFC neuron pairs often displayed central peaks in their crosscorrelograms, suggesting that their spikes are synchronized within tens or hundreds of milliseconds (see Results). To determine the time scale over which spikes are synchronized in the rat PFC during spatial working tasks and also to determine the time scale over which such correlated spikes are related to signal or noise correlation, the amount of correlated spikes was calculated for variable intervals of time lags in steps of $20 \mathrm{msec}$ between 20 and $500 \mathrm{msec}$, using the index of spike correlation, $I(t)$, defined as the following:

$$
I(t)=\log _{10}[S(t) / E],
$$

where $S(t)$ represents the rate of spikes synchronized with time lags between $t$ and $t-20 \mathrm{msec}$. One millisecond time lag ( $\pm 1 \mathrm{msec}$ interval around the zero lag) was omitted from the calculation because of the limited temporal resolution of successive spike detection with the current recording method (spikes occurring in two neurons within $1 \mathrm{msec}$ from each other were not detected with the current recording method, so they were not included in calculation of signal and noise correlation, either). $E$ is the expected level of synchronous firing based on the assumption that the two spike trains were independent Poisson processes. Therefore, positive (negative) values of $I(t)$ would indicate excessive (reduced) level of correlated spikes occurring within a particular range of time lags between a neuron pair. If the spike trains of the two neurons were stationary Poisson processes, $E$ would be simply the product of the mean firing rates. In the present study, however, the mean firing rates were modulated across the behavioral stages. Therefore, $E$ was defined as the following:

$$
E=\frac{\Delta T}{T} \sum_{\mathrm{i}} \lambda x_{i} \lambda y_{i} T_{i}
$$

where $\lambda x_{\mathrm{i}}$ and $\lambda y_{\mathrm{i}}$ are mean firing rates of cell $x$ and $y$ during stage $i$ (i.e., signal $i$ ), $T_{\mathrm{i}}$ is the duration of stage $i, \Delta T$ is the duration of the time step (38 msec for the first time step and $40 \mathrm{msec}$ for all others), and $T=\Sigma_{i} T_{\mathrm{i}}$. To improve the reliability of the results, only the units with $>200$ spikes were included in this analysis.

\section{RESULTS}

\section{Firing characteristics of neighboring neurons}

Of 131 and 68 pairs of single units that were recorded simultaneously with a single tetrode during the eight-arm maze and figure eight maze task, respectively, spatial firing rate maps of randomly selected six pairs for each task are shown in Figure 2. Many neighboring PFC neuron pairs displayed quite different relationships with respect to behavior during both tasks (Fig. 2). These results show qualitatively that most neighboring PFC neurons behave quite differently during spatial working memory tasks. 


\section{8-arm radial maze}
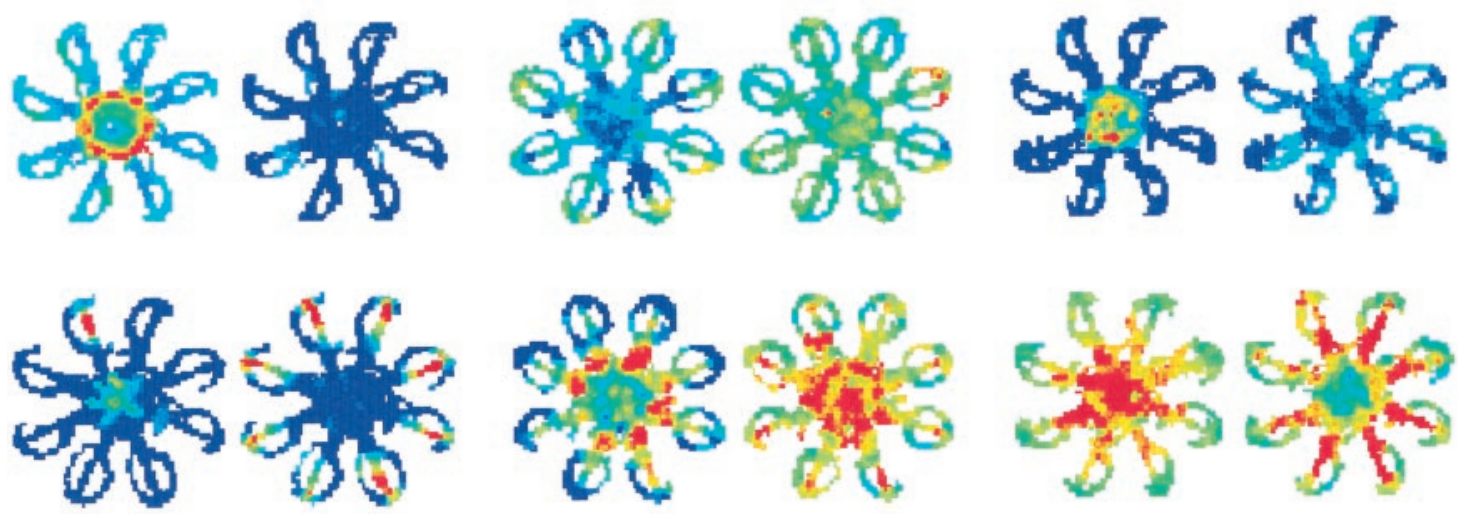

\section{Figure-8 maze}
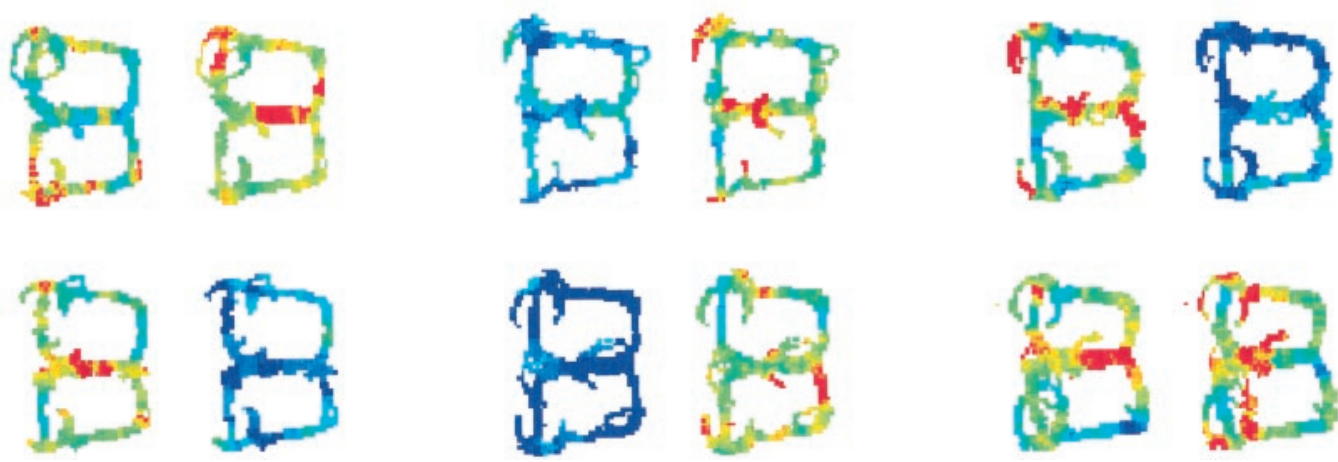

Figure 2. Spatial firing rate maps of simultaneously recorded PFC units. Each pair in the figure represents the spatial distribution of the firing rate (number of spikes per occupancy time) for a pair of simultaneously recorded PFC units. Red indicates maximum firing rates, which are different for each firing rate map, and dark blue indicates no firing. The lowest value for the maximum firing rate was set at $1 \mathrm{~Hz}$. Top, Spatial firing rate maps of six randomly selected pairs during the eight-arm radial maze task. The first unit in the second row is a rare unit that showed spatially biased firing; it was active in one arm but not in others. The maximum firing rates are (from left to right, top to bottom) $8,1,1,4.6,3.5,1,1.7,4.5,2.4,6.6,6.8$, and 5.4 Hz. Signal correlation of each unit pair is (from left to right, top to bottom) 0.190, $-0.066,-0.253,0.194,0.701$, and -0.086 . Bottom, Spatial firing rate maps of six randomly selected pairs during the figure eight maze task. The maximum firing rates are (from left to right, top to bottom) 1, 5.9, 1, 1.4, 1.8, 1, 2, 1, 1, 1.8, 1.7, and $1.2 \mathrm{~Hz}$. Signal correlation of each unit pair is (from left to right, top to bottom) $-0.484,0.106,0.698,-0.610,-0.262$, and -0.148 .

\section{Signal and noise correlation}

In this report, raw correlation coefficients for signal and noise correlation were subjected to Fisher's $z$ transformation (see Materials and Methods) for normalization as in previous studies ( $\mathrm{Zo}$ hary et al., 1994; Lee et al., 1998a), and the means and SEs of these $z$ transforms are reported (the average correlation coefficients before and after Fisher's $z$ transformation were similar). Overall, signal correlation during the eight-arm radial maze task was low $(0.203 \pm 0.061)$, and noise correlation was even lower $(0.061 \pm$ $0.018)$. Likewise, signal correlation was low $(0.124 \pm 0.058)$, and noise correlation was lower $(0.053 \pm 0.015)$ during the figure eight maze task, too (Fig. 3A). Below, we addressed the following issues: (1) whether these values of signal-to-noise correlation are statistically significant, (2) whether our measures of signal (i.e., mean firing rates across different behavioral epochs) were reliable, (3) whether low noise correlation could be attributable to a slow, parallel drift in the discharge rate of multiple neurons, (4) whether these results could be adversely affected by relatively low firing rates of PFC neurons, and (5) whether neuronal pairs with similar signals (i.e., high signal correlation) display higher noise correlation.

To evaluate the statistical significance, signal and noise correla- tion were recalculated after the temporal relationship between the two neurons was destroyed (see Materials and Methods). Correlation coefficients for signal and noise that were obtained after random shuffling of responses were $0.018 \pm 0.038$ and $0.013 \pm$ 0.010 for the radial maze task and $-0.058 \pm 0.046$ and $-0.011 \pm$ 0.010 for the figure eight maze task, respectively. Both signal and noise correlation in the original data were significantly higher than those of randomly shuffled data for the radial maze task ( $t$ test, $p<$ 0.01 and $p<0.05$, respectively) and the figure eight maze task ( $t$ test, $p<0.01$ and $p<0.001$, respectively).

Correlation coefficient calculated across the two halves of the behavioral stages was close to 1 (see Materials and Methods); it was $0.973 \pm 0.067$ for the radial maze task and $0.932 \pm 0.096$ for the figure eight maze task (Fig. 3). These values were substantially higher than the signal correlation between neighboring neurons, demonstrating that the observed low signal correlation is not attributable to weak or unreliable signals. Statistical analysis indicates that the new correlation calculated across the two substages was significantly higher than the original signal correlation $(t$ test, $p<0.001$ for both tasks).

Although we found statistically significant noise correlation among the neighboring PFC neurons, correlation coefficient itself 
A

Radial maze

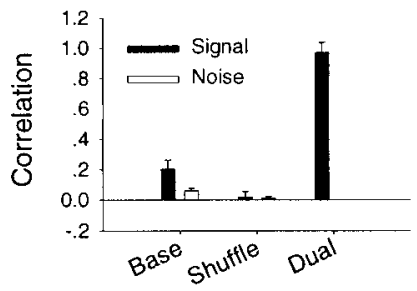

B

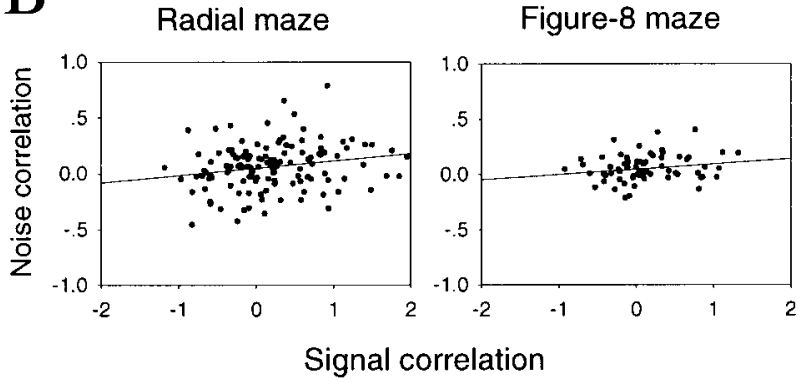

Figure 3. Correlated discharges of neighboring PFC neurons. A, Signal and noise correlation without manipulation (base), after random shuffling (shuffle), and between same neural responses that were divided into two data sets (dual) are shown for the eight-arm radial maze and the figure eight maze. Data are mean \pm SEM. Signal and noise correlation in this and subsequent figures indicate $z$ transforms of correlation coefficients. $B$, Relationship between signal correlation and noise correlation during the eight-arm radial maze and the figure eight maze task. The solid lines were determined by linear regression. Only the correlation during the eight-arm radial maze was statistically significant $(p<0.05)$.

does not reveal the time scale over which the neuronal noise is correlated. One can consider at least two different possibilities. For one, the discharge rates of multiple PFC neurons might fluctuate from trial to trial in synchrony. Another possibility is that the excitability of two neurons might drift slowly in the same direction over the course of the experiment ("yoked drift"; Zohary et al., 1994). To distinguish between these two alternatives, we performed the following control analysis. In this analysis, the original temporal relationship between the two spike trains was destroyed by shifting the responses of one neuron by one trial relative to the responses of the other neuron, and noise correlation was recalculated based on the responses in the remaining $\mathrm{N}-1$ trials. This would leave any slow yoked drift largely intact while destroying temporal relationship between two spike trains. The resulting noise correlation $(-0.006 \pm 0.017$ in the radial maze task and $0.005 \pm$ 0.012 in the figure eight maze task) was significantly lower than the original noise correlation ( $p<0.001$ in both tasks). Thus, it is not likely that the observed noise correlation was entirely attributable to the slow, yoked drift.

We also tested the possibility that the signal and noise correlation were adversely affected by low firing rates of some neurons. The regular spiking cells recorded in the present study have somewhat lower firing rates than those reported in other monkey studies (average firing rates were 2.2 and $2.3 \mathrm{~Hz}$ in the radial and figure eight mazes, respectively; fast-spiking cells fired at higher rates than $10 \mathrm{~Hz}$; Jung et al., 1998). In this analysis, we divided unit pairs into two groups. One group contained the unit pairs in which both units fired at $\geq 1 \mathrm{~Hz}$ (high rate group, $n=28$ in the radial maze and $n=12$ in the figure eight maze task). The other group contained unit pairs in which at least one unit fired at a rate $<1 \mathrm{~Hz}$ (low rate group, $n=130$ in the radial maze and $n=65$ in the figure eight maze task). In both tasks, neither the signal correlation nor the noise correlation differed significantly for the low and high rate groups. For the radial maze task, average signal correlations of the high rate and low rate groups were $0.162 \pm 0.146$ and $0.214 \pm 0.067$, respectively. Average noise correlations were $0.042 \pm 0.049$ and $0.066 \pm 0.019$. Similarly, for the figure eight maze task, average signal correlations were $0.102 \pm 0.174$ and $0.129 \pm 0.061$ for the high and low rate groups, respectively, whereas noise correlations were $0.069 \pm 0.054$ and $0.049 \pm 0.014$.

There was weak correlation between signal correlation and noise correlation in each task, suggesting that neuron pairs with relatively high signal correlation are likely to display high noise correlation as well (Fig. 3B). Correlation coefficients $(r)$ between signal correlation and noise correlation were 0.227 for the radial maze task and 0.190 for the figure eight maze task. The correlation between signal correlation and noise correlation was significant in the radial arm maze task $(p<0.01)$ but not in the figure eight maze task $(p=0.10)$. The higher $p$ value for the figure eight maze task may be attributable to a smaller number of pairs $(n=68)$ compared to the radial maze task $(n=131)$.

In their study of neurons in the inferior temporal cortex, Gawne and Richmond (1993) reported the magnitude of redundant signals using $R^{2}$ of linear regression, because they were interested in how reliably one can predict the signal of a neuron based on that of another neuron, regardless of the sign of correlation. In this report, we have concentrated on the values of correlation coefficient, because we were mainly interested in how similar the activities of neighboring PFC neurons might be. Nevertheless, when the correlation between signals of neighboring PFC neurons was expressed in terms of $R^{2}$, the average values were $0.248 \pm 0.023$ and $0.161 \pm$ 0.024 for the radial and figure eight maze tasks, respectively, which are similar to the values reported previously $(\sim 0.2$; Gawne and Richmond, 1993; Gawne et al., 1996). Gawne and Richmond (1993) also determined noise correlation by measuring $R^{2}$ of linear regression. The level of noise correlation quantified using the same method was $0.041 \pm 0.006$ and $0.016 \pm 0.003$ for the radial and figure eight maze tasks respectively, which are somewhat lower but not very different from the previously reported values $(\sim 0.05$; Gawne and Richmond, 1993; Gawne et al., 1996).

\section{Relationship between the two tasks}

Forty-two neuron pairs were recorded in both tasks. The relationship between signal correlations during the two tasks is shown in Figure $4 A$. There was only a weak relationship. The correlation coefficient was 0.094 , which was not significantly different from zero, suggesting that the degree to which two nearby PFC neurons carry similar signals in their discharge rates varies across behavioral tasks. Likewise, there was no significant relationship between noise correlations during the two tasks (Fig. 4B). Its correlation coefficient was 0.012 .

\section{Time scale of correlated spikes}

One hundred and four unit pairs (81 and 23 from the radial and figure eight maze task, respectively) that had $>200$ spikes in both units, were included in the cross-correlation analysis. Crosscorrelograms between the neighboring PFC neurons often displayed central peaks, but the width of these central peaks was rather variable. Some of them had relatively sharp peaks confined within $\pm 10 \mathrm{msec}$ (Fig. $5 A$ ), whereas others displayed broader peaks (Fig. $5 B$ ). Similarly, the population average for the index of spike correlation decreased continuously up to $\sim 500 \mathrm{msec}$ (Fig. 6), suggesting that in many neuron pairs, spikes were synchronized over a several hundred milliseconds more frequently than would be expected from two independent spike trains (see Materials and Methods).

To examine whether these correlated spikes contribute to signal correlation, noise correlation, or both, the following regression model was applied separately for each time lag:

$$
I(t)=\mathrm{a}_{\mathrm{o}}+\mathrm{a}_{1} r
$$

where $I(t)$ denotes the index of spike correlation with time lags between $t$ and $t-20 \mathrm{msec}$, and $r$ refers to signal (or noise) correlation. Because the population average for the index of spike correlation approached zero near the time lag of $500 \mathrm{msec}$, this analysis was applied up to $500 \mathrm{msec}$ of the time lag. For each regression line, the slope $\left(a_{1}\right)$ and the correlation coefficient were 


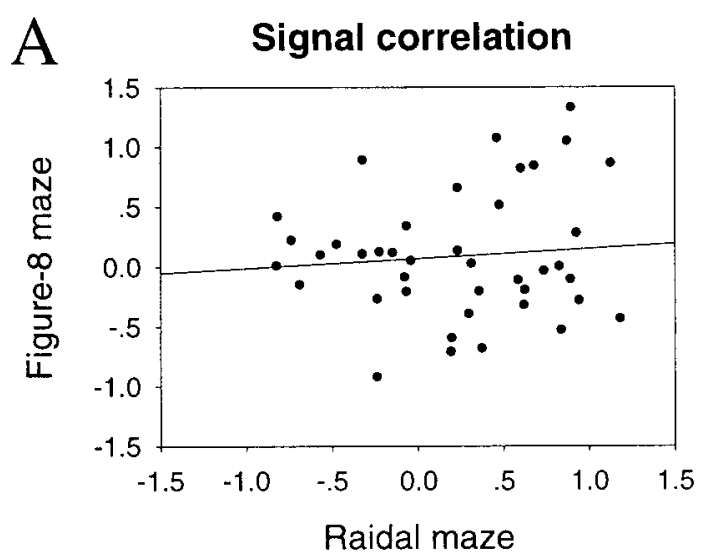

\section{B}

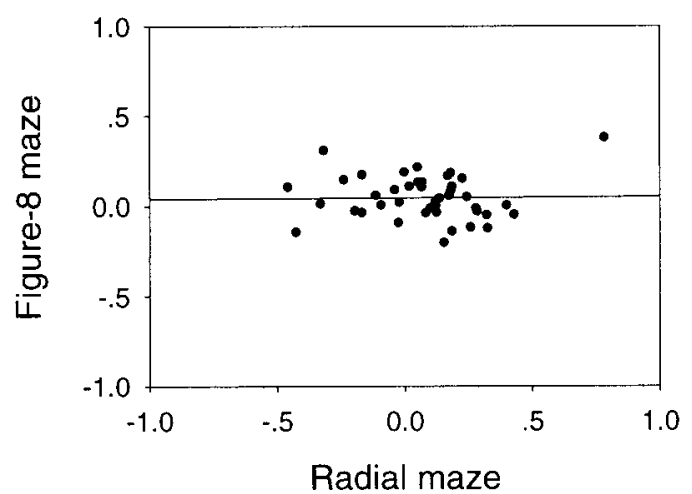

Figure 4. Relationship between the two tasks. $A$, Relationship between signal correlations during the eight-arm radial maze and the figure eight maze task. $B$, Relationship between noise correlations during the two tasks. The solid lines are results of linear regression. Neither relationship was statistically significant.

calculated. If the spikes underlying the signal (or noise) correlation were largely synchronized only over a short time scale, the slope of this regression line as well as the correlation coefficient would remain relatively small except for relatively small time lags. The slopes of the regression lines decreased slightly with the time lag, but they remained positive up to $500 \mathrm{msec}$ time lag (Fig. 7A). Similarly, correlation coefficient between the index of spike correlation and the signal (or noise) correlation did not decrease significantly as a function of time lag (Fig. $7 B$ ), suggesting that correlated spikes underlying the signal or noise correlation are broadly distributed in time.

As mentioned above, some of the neuron pairs in the present study displayed relatively sharp peaks in their cross-correlograms. To determine whether these more precisely synchronized spikes contribute to the signal or noise correlation, we first calculated the average values of the cross-correlograms within $\pm 500 \mathrm{msec}$, omitting the central $\pm 50 \mathrm{msec}$. Then, the rate of spikes synchronized within \pm 50 msec was divided by this baseline and log-transformed. Whereas the index of spike correlation measures the rate of spikes correlated in time with various time lags beyond the degree predicted by two independent spike trains, this new measure of synchronous spikes reflects whether spikes are more likely to be synchronized within a short interval $(<50 \mathrm{msec})$ than they are over a broad time scale (Lee et al., 1998a). There was only a weak relationship between this measure and the signal (and noise) correlation; correlation coefficient was 0.159 for signal correlation and 0.185 for noise correlation $(p>0.05)$. Similar to the results obtained with the index of spike correlation, these results also suggest that precisely synchronized spikes are not the major contributors to the overall correlation in the activities of PFC neurons.
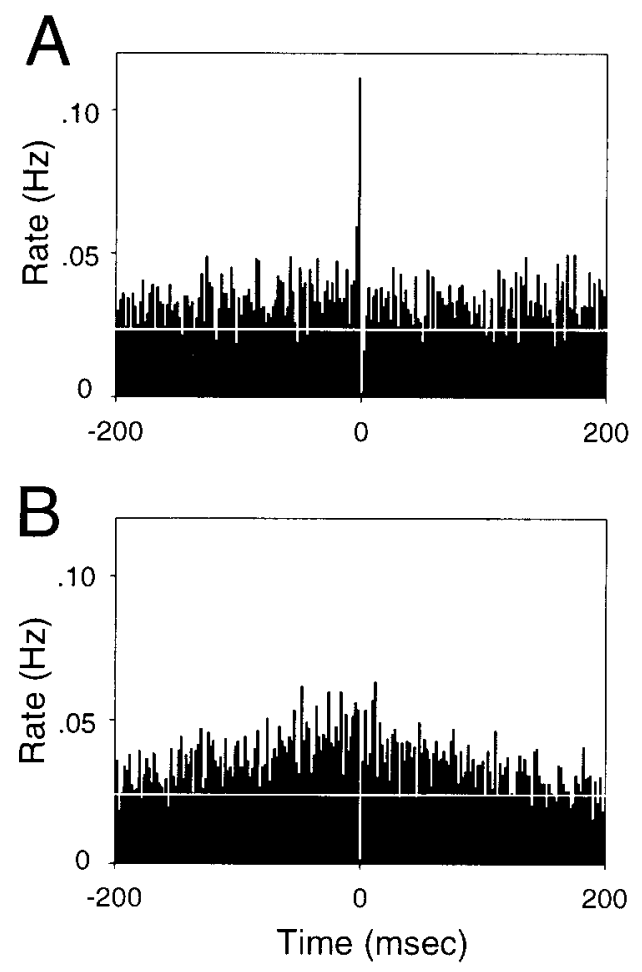

Figure 5. $A, B$, Two examples of cross-correlograms. The ordinate indicates firing rate. White horizontal lines indicate expected levels of synchronous firing under the assumption that two spike trains are independent Poisson processes.

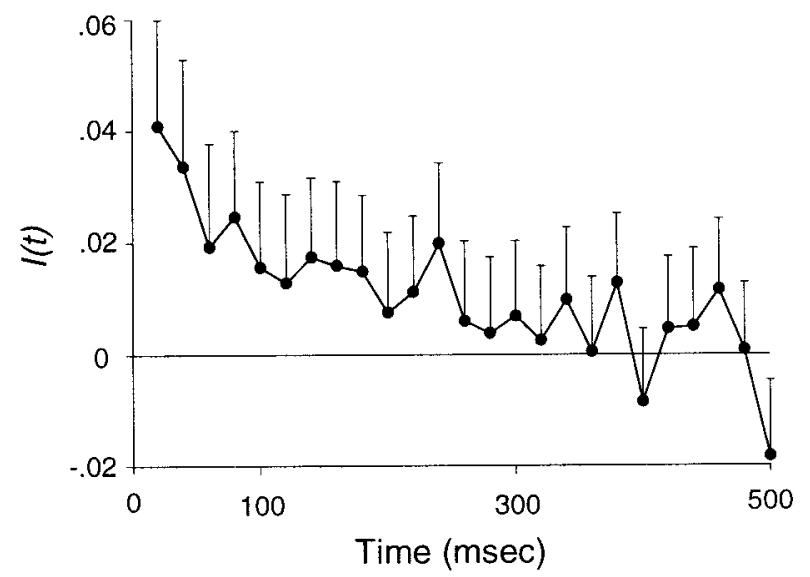

Figure 6. Population average of index of spike correlation, $I(t)$, which is defined as the rate of spikes synchronized with the time lags between $t$ and $t-20 \mathrm{msec}$, is shown for $t=20 \mathrm{up}$ to $500 \mathrm{msec}$ in $20 \mathrm{msec}$ steps. Error bars indicate SEM.

\section{DISCUSSION}

We examined patterns of correlation in the discharges of neighboring PFC neurons that were recorded with tetrodes during spatial working memory tasks. Three main observations were made in this study. First, behavioral correlates of neighboring PFC neurons were quite heterogeneous, and hence signal correlation was low. Second, noise correlation was even lower. Third, individual spikes underlying signal and noise correlation were only loosely correlated in time over a few hundred milliseconds, and precisely synchronized spikes play a minor role in generating correlated signal and noise among the neighboring PFC neurons.

\section{Signal correlation}

Compared to sensory cortex, it is less obvious what the activity of PFC neurons represents and therefore how "signal" should be 

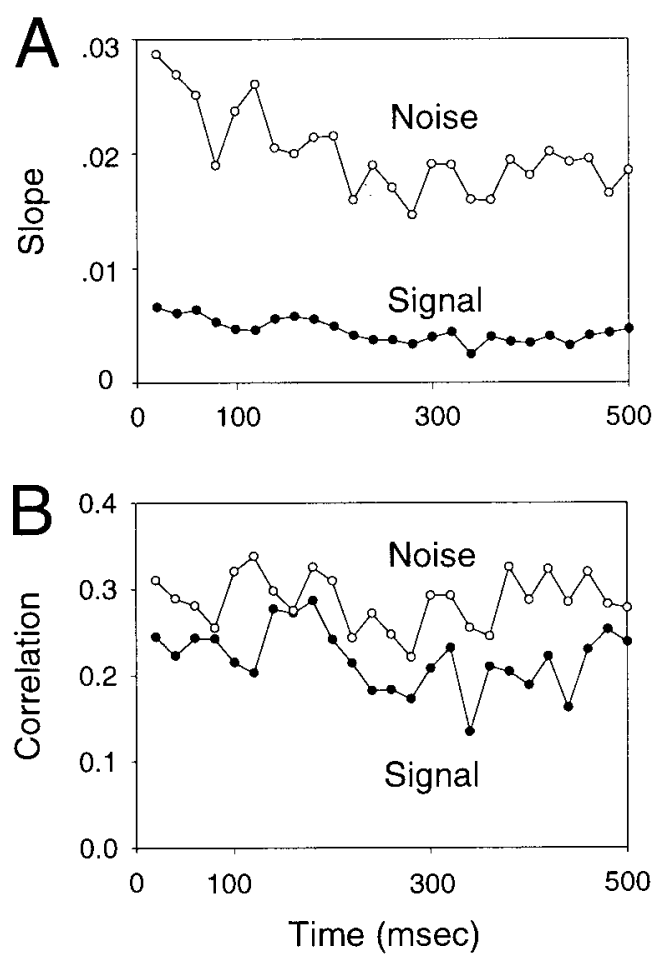

Figure 7. A, Slope of the regression line between signal (and noise) correlation and the index of spike correlation at different time lags. $B$, Correlation coefficient between signal (and noise) correlation and the index of spike correlation at different time lags.

defined. As described in our previous paper (Jung et al., 1998), however, not all but most units changed their firing rates in association with different stages on the eight-arm radial maze and figure eight maze. Many neurons showed activities that precisely turned on and off in association with particular stages of the tasks (Jung et al., 1998, e.g., their Fig. 6B), and these neurons were not directly related to immediate sensory inputs, ongoing behaviors, time, or other aspects of the tasks. This pattern of PFC activity can be contrasted with place-specific discharge patterns of hippocampal neurons on the eight-arm radial maze (Jung, 1995), which is consistent with the conclusions from the behavioral studies that the hippocampus encodes specific information about a given environment, whereas the PFC encodes general rules (i.e., structural features) of behavioral tasks (Winocur and Moscovitch, 1990). Thus, our definition of signals appears to be comparable to defining signals of sensory cortical neurons as mean discharge rates associated with different sensory stimuli (Gawne and Richmond, 1993) or signals of motor cortical neurons as mean discharge rates associated with movements in different directions (Lee et al., 1998a). However, because discharge characteristics of rat PFC neurons have been studied less extensively compared to sensory or motor cortical neurons, we cannot completely exclude the possibility that "signals" defined in the present study represent information processed by PFC neurons suboptimally.

Redundant information processing by a local pool of neurons is beneficial in many respects (see introductory remarks). On the other hand, redundancy limits the maximum information processing capacity. Because even a small amount of shared information sets a severe upper limit on the maximum capacity for information processing (Johnson, 1980; Gawne and Richmond, 1993), evolutionary pressure might have favored independent information processing among neighboring neurons between these opposing needs. Previous studies have shown that adjacent cortical neurons process only $\sim 20 \%$ shared information in the inferotemporal and primary visual cortex in response to two-dimensional visual stimuli (Gawne and Richmond, 1993; Gawne et al., 1996). The present study also indicates that information processed by neighboring PFC neurons is weakly correlated.

Since the classic studies of Mountcastle (1957) and Hubel and Wiesel (1962), it has been widely accepted that the cortical column represents an elementary unit of cortical computations and that neurons within the same column process similar information. Redundant signal processing within the same cortical column has also been reported in the monkey PFC (Rao et al., 1999). Recent physiological studies that used simultaneous recordings of multiple single units in primary visual cortex suggested, however, that response patterns of neurons within the same cortical column vary considerably (Gawne et al., 1996; Maldonado and Gray, 1996; DeAngelis et al., 1999). Combined with the present study, these results suggest that response characteristics of neurons within the same cortical column are not as homogeneous as previously assumed. Clearly, our results are inconsistent with a simplistic view that cortical columns consist of neurons processing identical signals. Currently, it is not entirely clear whether or not there is a general principle regarding the level of redundant signal processing within the cortical column. More quantitative studies on this matter are required in different cortical regions under various behavioral paradigms.

\section{Noise correlation}

Correlation in neuronal noise might be expected among adjacent neurons, because neighboring neurons tend to receive common inputs. Previous studies have shown that neighboring cortical neurons have weakly correlated noise (Gawne and Richmond, 1993; Zohary et al., 1994; Gawne et al., 1996; Lee et al., 1998a; Maynard et al., 1999). The present study also found a relatively low level of noise correlation among neighboring PFC neurons during spatial working memory tasks. Whether noise correlation is expressed with correlation coefficients (Zohary et al., 1994; Lee et al., 1998a; Maynard et al., 1999) or $R^{2}$ of linear regression (Gawne and Richmond, 1993; Gawne et al., 1996), average noise correlation was $\sim 0.1$ or lower in most cases. The highest reported value is only 0.21 (Maynard et al., 1999). Thus weakly correlated noise discharges among neighboring neurons may be a general principle of the cerebral cortex.

In general, PFC neurons changed their activity in association with different stages of the spatial working memory tasks, and behavioral correlates of neighboring neurons were largely independent. Nevertheless, compared to sensory or motor cortex where the nature of signals processed by cortical neurons is better understood (Zohary et al., 1994; Lee et al., 1998b), it is more difficult to evaluate how correlated noise affects signal averaging among the PFC neurons. Ultimately, the role of correlated noise in the PFC must be understood within the framework of information theory (Shannon and Weaver, 1949). In this study, however, there were small numbers of trials, and relatively long behavioral stages were required to include a large number of spikes. These features made it difficult to apply an information theoretic analysis (Maynard et al., 1999; Panzeri et al., 1999). For such analysis, appropriately designed behavioral tasks as well as a larger quantity of data would be required in future studies.

There was significant positive correlation between signal correlation and noise correlation during the radial maze task. If neighboring neurons have a substantial number of shared input connections, a positive correlation between signal correlation and noise correlation is likely to result, because both signal and noise correlation should be supported by common or reciprocal inputs. Correlation between signal correlation and noise correlation during the figure eight maze task was similar to that during the radial maze task, but not significant. There is no a priori reason to assume that the two tasks are different in terms of the way signal and noise are related. Therefore, it is likely that this lack of significant correlation is attributable to a smaller number of samples in the figure eight maze task $(n=63)$. 


\section{Relationship between the tasks}

Signal and noise correlation between neighboring PFC neurons during the eight-arm maze task did not predict those during the figure eight maze task. This is consistent with the previous results that showed no obvious relationship between behavioral correlates of identical PFC neurons during the two tasks (Jung et al., 1998). These results are not surprising considering that the PFC neural activities were not related to immediate sensory inputs or ongoing behaviors of the animal in this study. The present results are therefore in agreement with the previous conclusion that the two tasks are independently represented in the PFC (Jung et al., 1998). In addition, these results indicate that correlated discharges among neighboring neurons are not determined by local circuit properties alone, but rather are an outcome of interactions between a local circuit and afferent inputs.

\section{Synchronous firing}

Presumably, synchronous discharges of a neuron pair reflect common inputs or local connectivity between the neurons. The majority of excitatory connections arise locally in the neocortex (Peters, 1987). In addition, neighboring cortical neurons tend to share common afferent inputs (Eccles, 1981). This pattern of connections predicts synchronous discharges, redundant signal processing, and correlated noise discharges (Shadlen and Newsome, 1998), which were all observed in the present study. Therefore, one might expect that across a population of neurons, all of these three measures would be correlated with one another and that spikes underlying the observed signal and noise correlation are predominantly those that are synchronized over tens of milliseconds (Bair et al., 1999). On the other hand, when the rate of synchronous spikes was quantified relative to that predicted from two independent spike sources in the present study, it was correlated with both signal and noise correlation over a broad range of time lags up to $500 \mathrm{msec}$ (Fig. 6). Furthermore, when the rate of synchronous spike was calculated relative to the mean level in the cross-correlograms between 50 and $500 \mathrm{msec}$ time lags, its relationship with signal and noise correlation was not significant. These results suggest that spikes underlying correlated signal and noise are loosely synchronized and that precise spike synchronization is not the major factor in producing signal or noise correlation among PFC neurons.

\section{REFERENCES}

Bair W, Zohary E, Newsome WT (1999) Synchrony in cross-correlograms linked to spike count correlation in pairs of neurons in area MT. Soc Neurosci Abstr 29:276.

Bugbee NM, Goldman-Rakic PS (1983) Columnar organization of corticocortical projections in squirrel and rhesus monkeys: similarity of column width in species differing in cortical volume. J Comp Neurol 220:355-364.

DeAngelis GC, Ghose GM, Ohzawa I, Freeman RD (1999) Functional micro-organization of primary visual cortex: receptive field analysis of nearby neurons. J Neurosci 19:4046-4064.

Eccles JC (1981) The modular operation of the cerebral neocortex considered as the material basis of mental events. Neuroscience 6:1839-1856.

Gawne TJ, Richmond BJ (1993) How independent are the messages carried by adjacent inferior temporal cortical neurons? J Neurosci 13:2758-2771.

Gawne TJ, Kjaer TW, Hertz JA, Richmond BJ (1996) Adjacent visual cortical complex cells share about $20 \%$ of their stimulus-related information. Cereb Cortex 6:482-489.
Goldman-Rakic PS, Schwartz ML (1982) Interdigitation of contralateral and ipsilateral columnar projections to frontal association cortex in primates. Science 216:755-757.

Gray CM, Maldonado PE, Wilson M, McNaughton B (1995) Tetrodes markedly improve the reliability and yield of multiple single-unit isolation from multi-unit recordings in cat striate cortex. J Neurosci Methods 63:43-54.

Hubel DH, Wiesel TN (1962) Receptive fields, binocular interaction and functional architecture in the cat's visual cortex. J Physiol (Lond) 160:106-154.

Johnson KO (1980) Sensory discrimination: neural processes preceding discrimination decision. J Neurophysiol 45:1793-1815.

Johnson KO, Darian-Smith I, Lamotte C (1973) Peripheral neural determinants of temperature discrimination in man: a correlative study of responses to cooling skin. J Neurophysiol 36:347-370.

Jung MW (1995) Regional variations in the hippocampal place code. In: Emotion, memory and behavior: study of human and nonhuman primates (Nakajima T, Ono T, eds), pp 65-77. Tokyo: Japan Scientific Society.

Jung MW, Qin Y, McNaughton BL, Barnes CA (1998) Firing characteristics of deep layer neurons in prefrontal cortex in rats performing spatial working memory tasks. Cereb Cortex 8:437-450.

Lee D, Port NL, Kruse W, Georgopoulos AP (1998a) Variability and correlated noise in the discharge of neurons in motor and parietal areas of the primate cortex. J Neurosci 18:1161-1170.

Lee D, Port NL, Kruse W, Georgopoulos AP (1998b) Neuronal population coding: multielectrode recordings in primate cerebral cortex. In: Neuronal ensembles: strategies for recording and decoding (Eichenbaum H, Davis J, eds). pp 117-136. New York: Wiley.

Linsker R (1988) Self-organization in a perceptual network. Computer 21:105-117.

Maldonado PE, Gray CM (1996) Heterogeneity in local distributions of orientation-selective neurons in the cat primary visual cortex. Vis Neurosci 13:509-516.

Maynard EM, Hatsopoulos NG, Ojakangas CL, Acuna BD, Sanes JN, Normann RA, Donoghue JP (1999) Neuronal interactions improve cortical population coding of movement direction. J Neurosci 19:8083-8093.

Mountcastle VB (1957) Modality and topographic properties of single neurons of cat's somatosensory cortex. J Neurophysiol 20:408-434.

Panzeri S, Schultz SR, Treves A, Rolls ET (1999) Correlations and the encoding of information in the nervous system. Proc R Soc Lond Biol Sci 266:1001-1012.

Peters A (1987) Synaptic specificity in the cerebral cortex. In: Synaptic function (Edelman GM, Gall WE, Cowan WM, eds), pp 373-397. New York: Wiley.

Rao SG, Williams GV, Goldman-Rakic PS (1999) Isodirectional tuning of adjacent interneurons and pyramidal cells during working memory: evidence for microcolumnar organization in PFC. J Neurophysiol 81:1903-1916.

Recce ML, O'Keefe J (1989) The tetrode: an improved technique for multi-unit extracellular recording. Soc Neurosci Abstr 15:1250.

Rieke F, Warland D, de Ruyter van Steveninck RR, Bialek W (1997) Spikes: exploring the neural code. Cambridge, MA: MIT.

Shadlen MN, Newsome MW (1998) The variable discharge of cortical neurons: implications for connectivity, computation, and information coding. J Neurosci 18:3870-3896.

Shannon CE, Weaver W (1949) The mathematical theory of communication. Urbana, IL: University of Illinois.

Snedecor GW, Cochran WG (1989) Statistical methods. Iowa State University: Ames, IA.

van Kan PL, Scobey RP, Gabor AJ (1985) Response covariance in cat visual cortex. Exp Brain Res 60:559-563.

Wilson MA, McNaughton BL (1993) Dynamics of the hippocampal ensemble code for space. Science 261:1055-1058.

Winocur G, Moscovitch M (1990) Hippocampal and prefrontal cortex contributions to learning and memory: analysis of lesion and aging effects on maze learning in rats. Behav Neurosci 104:544-551.

Zohary E, Shadlen MN, Newsome WT (1994) Correlated neuronal discharge rate and its implications for psychophysical performance. Nature 370:140-143. 